\title{
Stress, anxiety and depression levels among nurses working at Hayatabad Medical Complex, Peshawar, Pakistan
}

\author{
Anayat Jan, Sehrish Naz, Ashfaq Ahmad, Shabnam Zamin
}

\section{Submitted}

May 21, 2021

Accepted

June 12, 2021

\section{Author Information}

Ms. Anayat Jan

Charge Nurse, Khyber

Teaching Hospital, Peshawar, Khyber Pakhtunkhwa,

Pakistan

\section{Ms. Sehrish Naz}

Lecturer, Institute of Nursing

Sciences, Khyber Medical

University, Peshawar, Khyber

Pakhtunkhwa, Pakistan

(Corresponding author)

Email:

sehrish.ins@kmu.edu.pk

Mr. Ashfaq Ahmad

Principal, Himalya College of

Nursing, Mardan, Khyber

Pakhtunkhwa, Pakistan

Ms. Shabnam Zamin

Demonstrator, Institute of

Nursing sciences, Khyber

Medical University,

Peshawar, Khyber

Pakhtunkhwa, Pakistan

Citation: Jan A, Naz S, Ahmad A, Zamin S. Stress, Anxiety and Depression levels among nurses working at Hayatabad Medical Complex, Peshawar, Pakistan. J Rehman Med Inst. 2021 Apr-Jun;7(2):12-5.

\section{ABSTRACT}

Introduction: Though mental disorders have remained global issues, their relationship with stressful life styles and occupations are a modern phenomenon. Anxiety and depression can become permanent components of personality, thereby affecting productivity and social activities.

Objective: To determine the stress, anxiety and depression levels among nurses working at Hayatabad Medical Complex, Medical Teaching Institution, Peshawar, Khyber Pakhtunkhwa, Pakistan.

Materials \& Methods: A cross-sectional descriptive study was conducted on 100 nurses by using convenient sampling technique at Hayatabad Medical Complex, Medical Teaching Institution, Peshawar, Khyber Pakhtunkhwa, Pakistan, over 6 months duration from March 2020 to August 2020. A valid, reliable and adopted DASS-21 Scale was used for data collection. The data were analyzed for descriptive statistics by SPSS version 22.0.

Results: Among all participants, $76 \%$ were female and $26 \%$ were male and have experience from 6 months to 20 years. More than half of the participants were highly affected by stress, anxiety and depression, and significant associations have been found between demographic variables, stress, anxiety and depression. The mean score of Stress, Anxiety and Depression was 16.74, 14.12 and 16.44 respectively.

Conclusion: In conclusion, health care settings should provide their employees a respectful, culture sensitive and supportive environment to single female staff where they feel safe and happy. Lastly, cross-sectional surveys should be conducted on these psychological problems to find out the key factors behind them.

Keywords: Stress, Anxiety, Depression, Nurses, Tertiary Care Hospital

The authors declared no conflict of interest. All authors contributed substantially to the planning of research, data collection, data analysis, and write-up of the article, and agreed to be accountable for all aspects of the work.

\section{INTRODUCTION}

Mental disorders have been observed as leading cause of infirmity around the globe. ${ }^{1}$ Disorders such as anxiety and depression prevail for long term in individual's personality and in turn compromise the productivity, ability, and any kind of physical activities. ${ }^{1}$ Stress in medical terms can be defined as the sensation of psychological and physical tension which can be developed from any situation or consideration that makes an individual feel frustrated, aggressive or restless. ${ }^{2}$ It is further divided into two types acute and chronic stress. ${ }^{2}$ Similarly, nursing is a profession of continuous stress with regards to health care concept. ${ }^{3}$ So, there are various reasons that cause stress and then resultantly stress gives birth to other mental disorders, among which the most common are depression and anxiety. ${ }^{3}$ It shows that each of the mental illness might have some association with each other. ${ }^{4}$ Quality care given by nurses to such intense mentally disordered patients helps them to achieve their day to day goals. Such patients feel depressed and are least focused and lose of concentration about anything happening around. ${ }^{4}$ Furthermore, in mental illness an individual experiences a part of anxious vitality and are incapable to unwind. Stress can come from two different sources. Inner source of stress comes from sickness or restorative strategy whereas outer source includes environment or societal situations. ${ }^{5}$ On the other hand, anxiety refers to the feelings of tension, panic thoughts, and some apparently changes cold shivering hands, feet and increased blood pressure. The individuals also experience tachycardia, excessive sweating, and trouble in breathing. ${ }^{6}$ According to the observation of WHO currently 322 million people are going through depression around the world. Half of the above figured population belongs to Western Pacific and South East Asian region. Most highlighted states include China and India. ${ }^{1}$ According to International literature, stress, depression, and anxiety are endemic in nursing career. A study showed 264 million people suffering from anxiety and clinical depression; since 2005, cases increased $15 \%$ by $2015 .^{7}$ According to another study conducted in Brazil and Italy, anxiety and depression increased in nursing profession with the 
ratio of $27 \%$ and $50 \%$ respectively. ${ }^{8}$ Results of a study from Saudi Arabia showed multiple mental disorders in nurses, accounted as $23 \%$ uneasiness disorder and $15 \%$ discouragement. ${ }^{9}$

In one of the study conducted in Iran, results showed that the result of stress were higher in male nurses whereas serious depression and anxiety was found higher in female nurses(10).A study in Peshawar conducted on stress among nurses which demonstrated that $82 \%$ of the nurses got stress with their work (11). Studies demonstrated that there is a solid affiliation among separate/ isolate/ single female nurses and medical issues such as anxiety, stress and depression prevails in them(12).An expansion of anxietyand stress is related with distinctive components such as age, work position and marital status(13). Nurses are responsible to look after their Patients and families(14).Stress, anxiety and depression can act as a barrier between quality of work and nurses endurance(15).The available published studies on the said topic are more than five years old so with new governmental reforms (Medical teaching institution system) of the tertiary care hospitals we became curious to replicate the study done in 2015(11) to find whether reforms have affected the nurses' performance positively or negatively so the aim of the study was to determine the stress, anxiety and depression level among nurses' working at Hayatabad Medical Complex Medical Teaching Institution (HMC,MTI), Peshawar, Pakistan.

\section{MATERIALS \& METHODS}

A cross-sectional descriptive study was conducted on nurses working in medical, surgical, ENT, Peads, Gynea\& Obs units of Hayatabad Medical Complex (HMC)who were registered with Pakistan Nursing Council and had more than 6 months experience. Study was conducted over a 6 months duration from March 2020 to August 2020.Nurses who were on long leave or absent from duties were excluded from the study. A nonprobability convenient sampling technique was used. Sample size was calculated through http://www.raosoft.com/samplesize.html, total population of medical and surgical unit nurses was 134 so keeping confidence interval 95\%, 5\% margin of error and assuming $50 \%$ response distribution, calculated sample size is 100.Permission was taken from Ethical review board of Institute of Nursing Sciences, Khyber Medical University and HMC administration prior to the commencement of data collection. A verbal as well as written inform consent was taken from the participants. Purpose and information's related to research were explained to the study participants. Anonymity to information's and confidentiality of the data were assured to the participants.

An adopted, validated and reliable DASS-21 questionnaire was used as a tool to measure stress, anxiety and depression level among nurses(16). DASS-21 comprised of 21 questions, 7 questions were for stress, 7 for anxiety and 7 for depression. Total score for normal to extremely severe depression was $0-28+$, for normal to extremely severe anxiety was $0-20+$ and for normal to extremely severe stress was 0-34+. Study variables were gender, work experience and marital status.

Data was analyzed using SPSS version 22.0. Descriptive statistics; frequency and percentages were measured for the demographical data. Frequency, percentages, mean and standard deviation was measured for other 21 questions. Data was presented through tables, figures and description.

\section{RESULTS}

According to the demographical variables, larger part of the participants was female $(74.0 \%$ ) (Table 1); $56 \%$ of the participants were unmarried; $61 \%$ of the participants had 1 to 5 years of experience, $28 \%$ had 6 to 10 years of experience, $6 \%$ had 11 to 15 years of experience and only $5 \%$ had 16 to 20 years of experience.

Table 1: Demographic data of the respondents $(n=100)$.

\begin{tabular}{|l|c|}
\hline \multicolumn{1}{|c|}{ Demographic Variables } & $\mathbf{f}=\%$ \\
\hline Gender & 26 \\
Male & 74 \\
Female & 56 \\
\hline Marital status & 44 \\
Unmarried & \\
Married & 61 \\
\hline Work experience (years) & 28 \\
$1-5$ & 06 \\
$6-10$ & 05 \\
$11-15$ & \\
$16-20$ & \\
\hline
\end{tabular}

Figure-1 shows a combined graph for stress, anxiety and depression level where the level of stress among $46 \%$ was normal whereas $11 \%$ were severe and $2 \%$ were extremely under-stress. Anxiety level was recorded normal in $22 \%$ whereas severe in $31 \%$ and extremely severe in $12 \%$ participants. Level of depression was found normal in $22 \%$, severe in $25 \%$ and extremely severe in $6 \%$ participants.

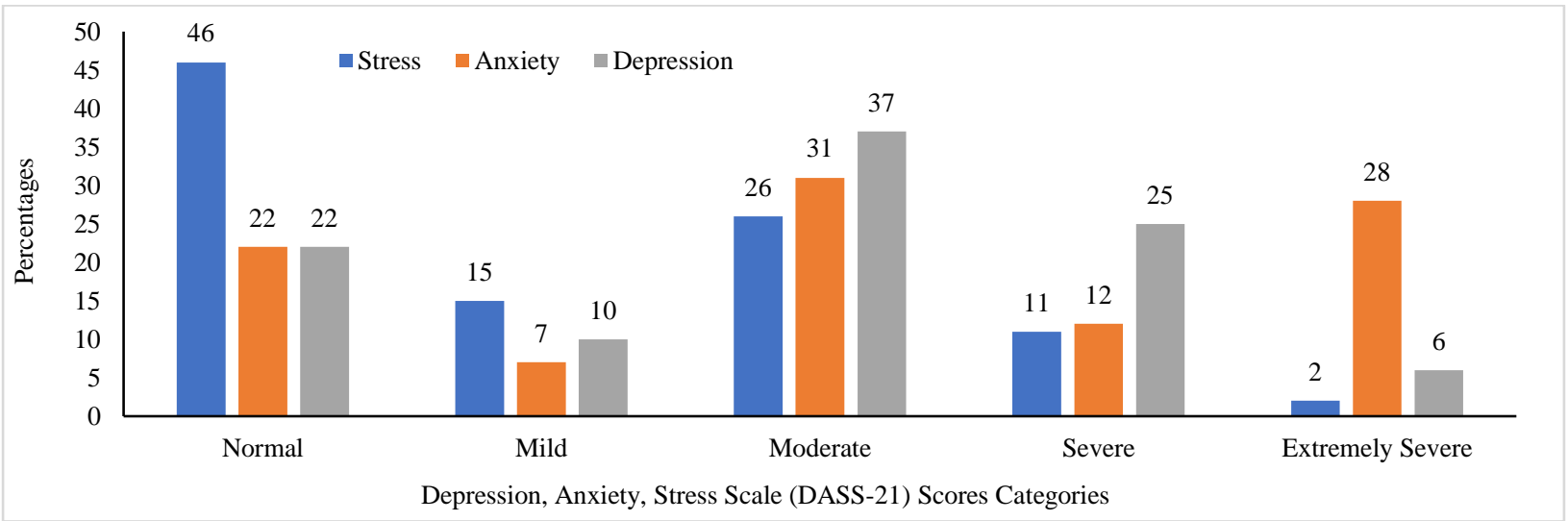

Figure 1: Combined Stress, Anxiety \& Depression levels of nurses (n=100). 
Mean score for stress was 16.74 , anxiety 14.12 and 16.44 for depression: in addition, anxiety and depression scores were near similar that showed that there is a positive association between anxiety and depression. According to demographic variables female and single participants were more anxious and depressed as compare to married participants. According to work experience the most juniors were more stressed, anxious and depressed, and these statements were verbally expressed by the participants.

\section{DISCUSSION}

This study was conducted to determine the level of stress, anxiety and depression among nurses. ${ }^{11}$ Results showed that $46 \%$ of the participants were stress free whereas $54 \%$ had mild to extremely severe stress. The results were much better than a previous study conducted in the same hospital and from other studies conducted in two other countries where $81 \%$ of the participants were stressful. A significant difference was found between 2015 and 2020 surveys which show that the nursing profession is getting changed from stressful life to stress free life since five year. ${ }^{11-13}$ Results of this study were also better than studies conducted in Iran and Tehran, which found that $57 \%$ of the participants were under mild to moderate stress. ${ }^{7,17}$ The current study found that $78 \%$ of the participants had mild to extremely severe anxiety and the same levels were found for depression. The study findings were not better than the studies conducted in China; where only $35.8 \%$ of the participants were depressed and $37.3 \%$ were anxious. ${ }^{6,18}$
According to a previous study, being female, unmarried, work experience, and place of service are additional risk factors for anxiety and depression. ${ }^{10} \mathrm{~A}$ similar situation was found in the present study where more of the junior participants stated that they were anxious and depressed. The anxiety and depression levels were fairly similar in the current study so it can be said that anxiety and depression have a positive relationship on the DASS21. In previous studies, it has been verified that participants in the anxiety subscale had a positive and significant relationship with the depression subscale. ${ }^{19-21}$

\section{CONCLUSION}

Majority of nurses working at the Hayatabad Medical Complex, Peshawar, Khyber Pakhtunkhwa had varying levels of stress, anxiety, and depression as assessed by the DASS-21; younger segments reported greater levels of stress and related anxiety and depression.

\section{RECOMMENDATIONS}

Hospital management should provide a respectful, culture sensitive, supportive, and conducive environment where nurses feel stress free and can provide quality care to their patients. The senior staff should help the juniors in adjusting to the new environment. Hospital management should conduct further surveys to find out the key factors behind the psychological issues of its employees.

\section{REFERENCES}

1. WHO. Depression and other common mental disorders: global health estimates. World Health Organization, 2017.

2. Penninx BW, Comijs HC. Depression and other common mental health disorders in old age. The epidemiology of aging: Springer; 2012. p. 583-98.

3. Lampert L. Depression in nurses: The unspoken epidemic. Minority Nurse. 2016;14.

4. dos Santos RP, da Silva Carvalho AR, Biederman B. Anxiety and depression among nursing professionals of a brazilian neonatal intensive care unit. Int J Dev Res. 2017;7(09):15431-5.

5. Abbas MAF, Abu Zaid L, Hussaein M, Bakheet KH, AlHamdan NA. Anxiety and depression among nursing staff at King Fahad medical city, Kingdom of Saudi Arabia. J Am Sci. 2012;8(10):778-94.

6. Cheung T, Yip PS. Depression, anxiety and symptoms of stress among Hong Kong nurses: a cross-sectional study. Int J Environ Res Pub Health. 2015;12(9):11072-100.

7. Zaher J, Vafaei M, Abianeh EE. Comparing depression, anxiety and stress among the Nurses in the critical care and internal surgical units at the selected hospitals of the Social Security
Organization of Tehran in 2016. Int J Med Res Health Sci. 2016;5:254-61.

8. Carugno M, Pesatori AC, Ferrario MM, Ferrari AL, Silva FJd, Martins AC, et al. Physical and psychosocial risk factors for musculoskeletal disorders in Brazilian and Italian nurses. Cadernos de saude publica. 2012;28:1632-42.

9. Saquib N, Zaghloul MS, Saquib J, Alhomaidan HT, Al-Mohaimeed A, AlMazrou A. Association of cumulative job dissatisfaction with depression, anxiety and stress among expatriate nurses in Saudi Arabia. J Nurs Manag. 2019;27(4):740-8.

10. Nia RG. Evaluating the degree of stress, anxiety, and depression among the emergency personnel in Kerman University of medical sciences. J Sci Res Essays. 2016;2(1):1-6.

11. Khan N, Anwar H, Sayed M. Prevalence of Stress Factors in Nurses in Leady Reading Hospital (LRH), Khyber Teaching Hospital (KTH) and Hayatabad Medical Complex (HMC) Hospitals, Peshawar, KPK. Int $\mathrm{J}$ Innov Res Dev. 2015;4(4).

12. Nor Idayu Z. The influence of leadership style, social support and workload toward stress among nurses: Universiti Utara Malaysia; 2016.

13. Johan S, Sarwar H, Majeed I. To identify the causes of stress among nurses working in intensive care unit of Ittefaq Hospital Lahore. Int $\mathrm{J}$ Soc Sci Manag. 2017;4(2):96-109.

14. Rajabi F, Jahangiri M, Molaeifar $H$ Honarbakhsh M, Farhadi P. Occupational stress among nurses and pre-hospital emergency staff: application of fuzzy analytic hierarchy process (FAHP) method. EXCLI J. 2018;17:808.

15. Machado DA, Figueiredo NMAd, Velasques LdS, Bento CAdM, Machado WCA, Vianna LAM. Cognitive changes in nurses working in intensive care units. Revista brasileira de enfermagem. 2018;71(1):73-9.

16. Samani S, Joukar B. A study on the reliability and validity of the short form of the depression anxiety stress scale (DASS-21). 2007.

17. Khodadadi E, Hosseinzadeh M, Azimzadeh R, Fooladi M. The relation of depression, anxiety and stress with personal characteristics of nurses in hospitals of Tabriz, Iran. Int J Med Res Health Sci. 2016;5(5):140-8. 
18. Shi L, Zhang D, Zhou C, Yang L, Sun T, Hao T, et al. A cross-sectional study on the prevalence and associated risk factors for workplace violence against Chinese nurses. BMJ Open. 2017;7(6):e013105.

19. Budiyanto B, Rattu A, Umboh J. Factors related to work stress of executive nurses in the icumentation room of Bethesda
General Hospital Gmim Tomohon. Public Health. 2019;8(3). Available from: https://ejournal.unsrat.ac.id/index.php/kes mas/article/view/23939.

20. Mohamedkheir RA, Amara ZM, Balla SA, Mohamed HAA. Occupational stress among nurses working in intensive care units in Public Hospitals of Khartoum
State, Sudan 2016. Am J Health Res. 2016;4(6):166-71.

21. Munyanziza T. Workplace stressors and coping strategies among the intensive care unit nurses at university teaching hospitals, Rwanda: University of Rwanda; 2019. 